\title{
Avaliação do entendimento da prescrição por idosos atendidos pelo Sistema Único de Saúde de Porto Alegre, RS, Brasil
}

\author{
Evaluation of prescription understanding by elderly attended by \\ Unified Health System, Porto Alegre, RS, Brazil
}

\author{
Mayara Becker Delwing', Daniela de Oliveira Carvalho², Paula Engroff ${ }^{3}$, Luísa Scheer Ely ${ }^{4}$, \\ Bruna Ferreira Máximo5 ${ }^{5}$ Fernanda Bueno Morrone ${ }^{6}$ \\ ${ }^{1}$ Farmacêutica Mestre em Assistência Farmacêutica pela Universidade Federal do Rio Grande do Sul, Farmacêutica na Unimed Vale do Taquari e Rio Pardo. \\ <mayarabdelwing@gmail.com> \\ 2 Farmacêutica, Mestre em Medicina e Ciências da Saúde, PUCRS. < danielacarvalho_farm@yahoo.com.br> \\ ${ }^{3}$ Farmacêutica, Doutora em Gerontologia Biomédica, Programa de Pós-Graduação em Gerontologia Biomédica, Instituto de Geriatria e Gerontologia, PUCRS \\ <paula.engroff@pucrs.br> \\ ${ }_{4}^{4}$ Farmacêutica, Doutora em Gerontologia Biomédica, Professora da Univates, Lajeado, Rio Grande do Sul. <luisa_ely@yahoo.com.br> \\ ${ }^{5}$ Farmacêutica, Faculdade de Farmácia, PUCRS. < bru.maximo@yahoo.com.br> \\ ${ }^{6}$ Farmacêutica, Doutora em Bioquímica, Professora da Faculdade de Farmácia e dos Programas de Pós-Graduação em Medicina e Ciências da Saúde e do \\ Mestrado Profissional em Biotecnologia Farmacêutica - PUCRS. <fernanda.morrone@pucrs.br>
}

\section{ARTICLE INFO}

\section{Article history}

Received: 20/07/2017

Accepted: 18/10/2017

Correspondent Author Fernanda Bueno Morrone Av. Ipiranga, 6681 - Pr. 12, sala 110 90619-900 Porto Alegre, RS, Brasil

$<$ fernanda.morrone@pucrs.br>

(C) 2017 All rights reserved

\section{Editors}

Alfredo Cataldo Neto

Paula Engroff

\begin{abstract}
RESUMO
OBJETIVO: Este estudo teve como objetivo avaliar o uso dos medicamentos e correlacionar o entendimențo da prescrição médica com o controle do perfil lipídico e pressão arterial (PA) de idosos. MÉTODOS: Tratou-se de um estudo observacional, transversal, quantitativo que avaliou 97 prontuários de idosos, atendidos pelo Sistema Único de Saúde, no ambulatório do Instituto de Geriatria e Gerontologia (IGG) e no Centro de Extensão Universitária (CEU) Vila Fátima, Porto Alegre/RS, de 2008 a 2009. RESULTADOS: Os resultados permitiram constatar que a falta de entendimento pode estar relacionada à progressão da idade da população estudada. $\bigcirc$ entendimento da prescrição por pacientes atendidos no Ambulatório do IGG foi maior quando comparado aos idosos que frequentavam o CEU $(p<0,05)$. Os parâmetros de PA e dislipidemia não estão correlacionados diretamente ao entendimento da prescrição. CONCLUSOES: Deste modo, é necessário um trabalho voltado para o acompanhamento farmacoterapêutico aos pacientes idosos de modo a melhorar a adesão da terapia nesta população.
\end{abstract}

PalaVRas-ChaVe: idoso; adesão à medicação; Sistema Único de Saúde.

\begin{abstract}
AIMS: This article aimed to evaluate the use of drugs and to correlate the understanding of the prescription with the elderly lipid profile and blood pressure. METHODS: It was an observational, cross-sectional, quantitative study, which evaluated 97 records of the elderly outpatient from Single Health System of the Institute of Geriatrics and Gerontology (IGG) and the University Extension Center (UEC) Villa Fatima, Porto Alegre/RS from 2008 to 2009. RESULTS: The results revealed that the lack of understanding might be related to the progression of age of the population. The prescription understanding of the patients attending the IGG was higher when compared to the elderly who attended the UEC $(p<0.05)$. The parameters of blood pressure and dyslipidemia are not directly correlated to the understanding of the prescription. CONCLUSIONS: Thus, it is necessary to work towardsfollowing of pharmacotherapy from elderly patients to improve therapy adhesion in this population.

KEYWORDS: aged; medication adherence; Unified Health System.
\end{abstract}




\section{INTRODUÇÃO}

O uso racional de medicamentos tem sido reconhecido como uma parte importante das políticas públicas de saúde. A Organização Mundial de Saúde (OMS) estabeleceu como seu grande desafio a melhoria na racionalidade do uso de medicamentos. ${ }^{1} \mathrm{O}$ uso racional implica no recebimento pelo paciente de medicamentos apropriados para suas condições clínicas, em doses e períodos adequados a suas necessidades individuais, além da oferta de tratamentos a um preço acessível. É preciso que se dispense em condições ótimas, com a necessária orientação e responsabilidade, e, que se cumpra o regime terapêutico já prescrito, da melhor maneira possível. ${ }^{2,3}$

Importantes mudanças têm ocorrido no mundo, assim como na sociedade brasileira e gaúcha, especialmente com o aumento significativo da população idosa, e um maior consumo de medicamentos, fazendo-se necessária uma nova reavaliação nas condições de saúde desta população. 4,5

Mais de $85 \%$ dos idosos no Brasil têm pelo menos uma enfermidade crônica e cerca de 15\% apresentam até cinco dessas doenças, como hipertensão e diabetes. Esses problemas crônicos são responsáveis por afetar a funcionalidade de órgãos e tecidos, que já estão comprometidos pelo processo de envelhecimento, e são acompanhados por uma maior demanda pelos serviços de saúde. A prescrição de medicamentos é a intervenção terapêutica mais realizada por clínicos e predispõe a população idosa aos riscos da prática de polifarmácia, definida como o uso de cinco ou mais medicamentos ou de pelo menos um medicamento desnecessário. Esta prática ocorre, muitas vezes devido a falta de uma revisão regular do regime terapêutico pelos profissionais de saúde e a sua consequência, especialmente no idoso, é grave podendo ser até fatal devido às alterações nos processos farmacocinéticos e farmacodinâmicos, que interferem na metabolização dos fármacos podendo ser mais frequentemente tóxicos. $., 7,8,9,10$

Estima-se que $23 \%$ da população consome $60 \%$ da produção nacional de medicamentos, sendo os idosos os principais consumidores. Destes, mais de $80 \%$ fazem uso de no mínimo um medicamento diariamente. A prescrição e o uso inadequado dos medicamentos podem resultar em efeitos indesejáveis, levando a internações e elevando o custo dos serviços de saúde. . $^{8,11}$

Além das peculiaridades da farmacocinética e farmacodinâmica desta faixa etária, deve-se considerar na prescrição o custo e o grande número de medicamentos prescritos, bem como a falta de adesão a terapia devido a dificuldades de compreensão da mesma, diminuição da acuidade visual e o esquecimento, comum nesta faixa etária, especialmente devido ao comprometimento cognitivo. Estudos mostram que a falta de entendimento da prescrição, pode ser decorrente da carência de informações e explicações tanto da terapia como da patologia do paciente, levando a não compreensão das terminologias utilizadas pelo prescritor. $^{8,12,13}$

Segundo a OMS, ${ }^{14}$ a adesão a tratamentos está relacionada a fatores determinantes, como: a severidade dos sintomas, nível de incapacidade (física, social, psicológica), taxa de progressão, severidade da doença e existência de comorbidades. Um dos fatores mais claramente relacionados envolve características da terapia medicamentosa e seu nível de complexidade, evidenciando que o número de medicamentos apresenta importante efeito sobre a adesão. ${ }^{8}$ Com isso, a não adesão ao tratamento pode agravar o quadro de saúde do idoso ou até mesmo levar ao aparecimento de complicações, refletindo nos resultados dos exames laboratoriais.

Valores de pressão arterial e perfil lipídico alterados, bem como a presença de diabetes mellitus são fatores de risco fortemente associados a eventos cardiovasculares tais como, doença arterial coronariana, doença cerebrovascular e insuficiência cardíaca. No Brasil, 27,4\% dos óbitos em 2003, foram decorrentes de doenças cardiovasculares, que poderiam ser prevenidas ou adiadas através do controle desses fatores de risco. ${ }^{15,16,17}$ A prevalência de brasileiros adultos com Hipertensão Arterial Sistêmica (HAS) é de aproximadamente 20\%, elevando-se a $70 \%$ em idosos. ${ }^{18}$

Portanto, este estudo teve como objetivo avaliar o uso dos medicamentos prescritos, o controle da pressão arterial e dislipidemia, bem como correlacioná-los com o entendimento da prescrição medicamentosa pela população idosa atendida pelo Sistema Único de Saúde (SUS).

\section{MÉTODOS}

\section{Delineamento do estudo}

Estudo observacional,transversal e quantitativo que teve como base a análise retrospectiva de dados fornecidos pelo Laboratório de Bioquímica e Genética Molecular e Parasitologia - IGG, que abrangeu o período de Maio de 2008 a Setembro de 2011, através de uma amostra de conveniência. Foi considerado o entendimento da prescrição médica pelos idosos atendidos pelo SUS obtido através de um questionário com questões referentes à prescrição, feitas no ambulatório do IGG e no CEU-Vila Fátima na cidade de Porto Alegre/RS. Posteriormente, analisaram-se 
os prontuários desses pacientes entrevistados, considerando os exames laboratoriais mais recentes, assim como a medida de pressão arterial da última consulta, diagnóstico do paciente e medicamentos prescritos.

Foram incluídos pacientes com idade superior a 60 anos, de ambos os sexos e diferentes classes sociais, que obtiveram escore satisfatório no Miniexame do Estado Mental e que possuíam a prescrição no momento da entrevista. Foram excluídos os pacientes que não tinham prontuário e os que não apresentavam medida de pressão arterial.

\section{Coleta de dados}

Os instrumentos utilizados para a coleta de dados foram: um questionário, previamente validado ${ }^{19}$,sobre o conhecimento da terapia medicamentosa utilizada e sobre a percepção do cuidado farmacêutico recebido pelos idosos no momento do recebimento dos medicamentos e prontuários do Ambulatório do IGG e do CEU-Vila Fátima. Foi possível identificar o perfil de utilização de medicamentos pelos idosos mediante dados de seguimento farmacoterapêutico contendo: dados de identificação do paciente, tempo de utilização dos medicamentos, patologia(s), medicamentos utilizados e posologia.

Ademais, avaliou-se o estado mental dos idosos mediante o Mini Exame do Estado Mental (MEEM) ${ }^{20}$, o qual consiste em um questionário com cinco domínios: orientação, memória imediata, atenção e cálculo, evocação e linguagem. $\mathrm{O}$ avaliador marca um ponto para cada resposta correta, com a pontuação máxima atingindo 30 pontos. Pontos de corte de acordo com a escolaridade em adultos brasileiros: analfabeto $=15$; um a 11 anos $=22$ e com escolaridade superior a 11 anos $=27 . .^{21}$

Para a identificação das substâncias a partir do nome comercial, utilizou-se o Dicionário de Especialidades Farmacêuticas 2009/201022. Os fármacos prescritos foram classificados segundo o Sistema de Classificação Anatômico Terapêutico e Químico (ATC). ${ }^{23}$

\section{Medidas de pressão arterial e colesterol}

Para classificar os valores de pressão arterial dos pacientes que mantinham tratamento farmacológico, como controlado e não controlado foram utilizadas as Diretrizes Brasileiras de Hipertensão Arterial ${ }^{24}$, que estima como referência valores inferiores a $140 \mathrm{mmHg}$ de pressão sistólica e inferiores a $90 \mathrm{mmHg}$ de pressão diastólica para os pacientes em tratamento, e em casos especiais, como pacientes diabéticos, valores inferiores a $130 \times 80 \mathrm{mmHg}$, devido ao risco cardiovascular aumentado.

A classificação da dislipidemia, em que foram analisados os valores de perfil lipídico (colesterol total, HDL-colesterol, LDL-colesterol e triglicerídeos) foi feita com base nas Diretrizes Brasileiras Sobre Dislipidemias e Diretriz de Prevenção da Aterosclerose do Departamento de Aterosclerose da Sociedade Brasileira de Cardiologia, que recomenda valores de colesterol total menor que $200 \mathrm{mg} / \mathrm{dL}$; HDL-colesterol maior que $40 \mathrm{mg} / \mathrm{dL}$; LDL-colesterol menor que $130 \mathrm{mg} / \mathrm{dL}$ e triglicerídeos menor que $150 \mathrm{mg} / \mathrm{dL}$. Consideraram-se as metas recomendadas a pacientes que apresentam fatores de risco, como por exemplo, a idade ( $\geq 45$ anos para homens e $\geq 55$ anos para mulheres)..$^{24}$

Este estudo foi aprovado pelo Comitê de Ética em Pesquisa da Pontifícia Universidade Católica do Rio Grande do Sul, conforme parecer número 08/04188. Os pacientes que participaram do estudo assinaram o Termo de Consentimento Livre e Esclarecido.

\section{Avaliação das Interações Medicamentosas}

As interações medicamentosas foram determinadas utilizando o programa Micromedex ${ }^{\circledR} 1.0$ Healthcare Series e tabuladas, recebendo cada interação um número. As interações foram classificadas em categorias de acordo com a escala de gravidade, em moderada (recomenda-se evitar a administração a menos que o benefício da co-administração supera o risco para o paciente), e gravidade alta (deve-se evitar a co-administração).

\section{Análise Estatística}

Os dados foram tabulados em Excel e analisados através do programa SPSS 17.0. A diferença entre dados de pacientes estudados foi avaliada pelos testes estatísticos qui-quadrado, Teste $t$ de Student e ANOVA.

\section{RESULTADOS}

Foram analisados 97 prontuários obtidos a partir das entrevistas realizadas com idosos atendidos pelo SUS, dos quais, 67 eram do Ambulatório do IGG e 30 do CEU-Vila Fátima, PUCRS. Do total de pacientes estudados, $83,5 \%$ eram do sexo feminino e $16,5 \%$ do sexo masculino. Em relação ao entendimento da prescrição, o sexo masculino apresentou entendimento de $62,5 \%$, enquanto que no sexo feminino foi de $56,8 \%$ (Tabela 1).

A idade dos pacientes analisados variou de 60 a 94 anos, compreendendo 39,2\% com idade entre 60 a 69 anos, $44,3 \%$ entre 70 a 79 anos, e 16,5\% com idade maior ou igual a 80 anos, sendo o entendimento dos pacientes da última faixa etária de apenas 50,0\%. 
Quando considerado o local de atendimento dos pacientes, o entendimento da prescrição por aqueles que frequentaram o Ambulatório do IGG foi de 65,7\%, já no CEU-Vila Fátima foi de somente 40,0\% mostrando uma diferença estatisticamente significativa $(\mathrm{p}=0,018)$ (Tabela 1$)$.

Tabela 1. Características da população estudada e avaliação da frequência de entendimento $(\mathrm{n}=97)$

\begin{tabular}{lccc}
\hline Características & $\begin{array}{c}\text { População } \\
\text { n (\%) }\end{array}$ & $\begin{array}{c}\text { Frequência de } \\
\text { entendimento } \\
(\%)\end{array}$ & $\mathbf{p}$ \\
$\begin{array}{l}\text { Sexo } \\
\text { Masculino }\end{array}$ & $16(16,5)$ & 62,5 & \\
$\quad$ Feminino & $81(83,5)$ & 56,8 & $\mathbf{0 , 6 7 3}$ \\
Faixa etária & & & \\
60-69 anos & $38(39,2)$ & 57,9 & \\
$70-79$ anos & $43(44,3)$ & 60,5 & $\mathbf{0 , 7 6 9}$ \\
$\geq 80$ anos & $16(16,5)$ & 50,0 & \\
Local & & & \\
IGG & $67(69,1)$ & 65,7 & $* 0,018$ \\
CEU - Vila Fátima & $30(30,9)$ & 40,0 & \\
\hline
\end{tabular}

$\mathrm{p}=$ Nível de significância a partir de comparação estatística por teste qui-quadrado. $*(\mathrm{p}<0,05)$.

Do total de pacientes estudados que possuem a pressão arterial (PA) controlada 36,7\% entendem a prescrição e 34,3\% não entendem. Já em relação ao controle da dislipidemia, daqueles que mantêm o perfil lipídico dentro dos limites considerados adequados, $62,0 \%$ entendem a prescrição, enquanto que $44,7 \%$ não entendem. Quando analisado cada local de atendimento, em relação aos que apresentam perfil lipídico controlado no Ambulatório do IGG, 68,4\% entendem a prescrição e 45,0\% não a entendem (Tabela 2).

Tabela 2. Relação do controle dos níveis pressóricos e da dislipidemia com o entendimento da prescrição $(\mathrm{n}=97)$

\begin{tabular}{|c|c|c|}
\hline Grupo & $\begin{array}{c}\text { Controle da } \\
\text { pressão arterial } \\
\text { n (\%) }\end{array}$ & $\begin{array}{l}\text { Controle da } \\
\text { dislipidemia } \\
\text { n (\%) }\end{array}$ \\
\hline $\begin{array}{l}\text { Ambulatório IGG } \\
\text { Entendem a prescrição } \\
\text { Não entendem a prescrição } \\
\text { p }\end{array}$ & $\begin{array}{c}13(34,2) \\
06(33,3) \\
\mathbf{0 , 9 4 8}\end{array}$ & $\begin{array}{c}26(68,4) \\
09(45,0) \\
\mathbf{0 , 0 8 3}\end{array}$ \\
\hline $\begin{array}{l}\text { Vila Fátima } \\
\text { Entendem a prescrição } \\
\text { Não entendem a prescrição } \\
\text { p }\end{array}$ & $\begin{array}{c}05(45,5) \\
06(35,3) \\
\mathbf{0 , 5 9 1}\end{array}$ & $\begin{array}{c}05(41,7) \\
08(44,4) \\
\mathbf{0 , 8 8 0}\end{array}$ \\
\hline $\begin{array}{l}\text { Total } \\
\text { Entendem a prescrição } \\
\text { Não entendem a prescrição } \\
\text { p }\end{array}$ & $\begin{array}{c}18(36,7) \\
12(34,3) \\
\mathbf{0 , 8 1 7}\end{array}$ & $\begin{array}{c}31(62,0) \\
17(44,7) \\
\mathbf{0 , 1 0 7}\end{array}$ \\
\hline
\end{tabular}

$\mathrm{p}=$ nível de significância a partir de comparação estatística por teste qui-quadrado. $(\mathrm{p}<0,05)$
Como demonstrado na Tabela 3, a média de medicamentos utilizados pelo grupo estudado foi de 6,0 $\pm 2,4$ fármacos, sendo $6,45 \pm 2,5$ no Ambulatório do IGG e 4,9 $\pm 1,8$ no CEU-Vila Fátima, mostrando uma diferença estatisticamente significativa $(\mathrm{p}=0,040)$.

Tabela 3. Número de medicamentos utilizados pelos idosos $(\mathrm{n}=97)$

\begin{tabular}{lcc}
\hline Fator & $\begin{array}{c}\text { Número de medicamentos } \\
(\mathbf{m} \pm \mathbf{d p})\end{array}$ & $\mathbf{p}$ \\
\hline Sexo & & \\
$\quad$ Masculino & $6,4 \pm 2,3$ & $\mathbf{0 , 4 1 6}$ \\
$\quad$ Feminino & $5,9 \pm 2,5$ & \\
Faixa etária & & \\
60-69 anos & $5,7 \pm 2,9$ & $\mathbf{0 , 5 9 6}$ \\
$70-79$ anos & $6,0 \pm 2,2$ & \\
$\geq 80$ anos & $6,4 \pm 1,9$ & \\
Local & & \\
IGG & $6,45 \pm 2,5$ & \\
$\quad$ CEU-Vila Fátima & $4,90 \pm 1,8$ & \\
Total & $6,0 \pm 2,4$ & \\
\hline
\end{tabular}

$\mathrm{p}=$ Nível de significância a partir de comparação estatística por teste $t$ de Student e ANOVA. * $(\mathrm{p}<0,05)$

Os medicamentos mais utilizados para o controle da pressão arterial foram os diuréticos tiazídicos $(62,9 \%)$, seguido dos inibidores da enzima conversora da angiotensina (IECA) (51,5\%), betabloqueadores $(36,1 \%)$ e bloqueadores dos canais de cálcio $(24,7 \%)$ (Figura 1). Para o tratamento medicamentoso hipolipêmico, as estatinas foram utilizadas por todos os pacientes $(100,0 \%)$.

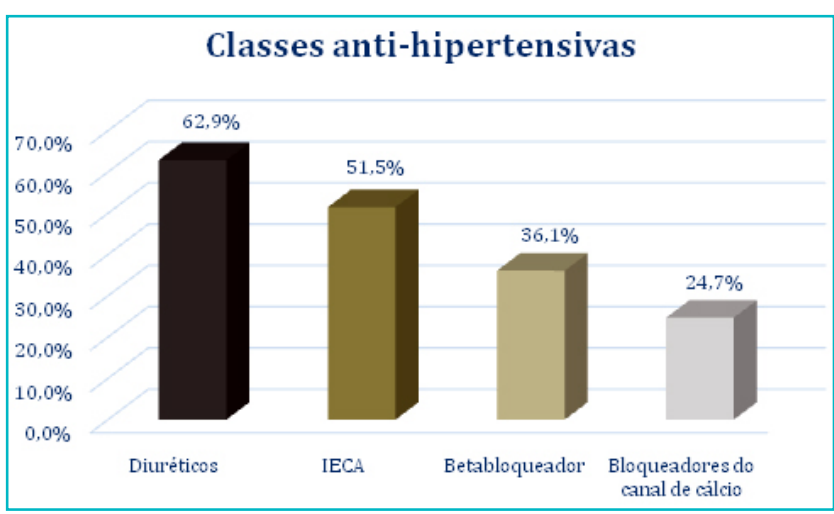

Figura 1. Classes de anti-hipertensivos mais utilizados pelos idosos estudados $(n=84)$

Foram identificadas 50 diferentes interações medicamentosas consideradas pela escala de gravidade, altas (17) ou moderadas (33). Do total de idosos estudados, 52 apresentavam ao menos uma interação 
Tabela 4. Interações medicamentosas mais frequentes, baseando-se nas prescrições da população idosa estudada $(\mathrm{n}=97)$

\begin{tabular}{|c|c|c|c|}
\hline Interações medicamentosas & Gravidade & Possível efeito & $\begin{array}{c}\text { Frequência } \\
\text { n (\%) }\end{array}$ \\
\hline Hipoglicemiante oral (biguanida) $\times$ Betabloqueador & Moderada & $\begin{array}{c}\text { Hiperglicemia, hipoglicemia } \\
\text { ou Hipertensão }\end{array}$ & $5(7,2)$ \\
\hline Bloqueadores dos canais de cálcio $\times$ Betabloqueador & Moderada & $\begin{array}{l}\text { Hipotensão } \\
\text { e/ou Bradicardia }\end{array}$ & $5(7,2)$ \\
\hline Hidroclorotiazida (diurético tiazídico) $\times$ Propranolol (betabloqueador) & Moderada & $\begin{array}{l}\text { Hiperglicemia } \\
\text { e/ou Hipertrigliceridemia }\end{array}$ & $4(5,1)$ \\
\hline
\end{tabular}

*IECA: inibidores da enzima conversora da angiotensina.

medicamentosa, sendo um deles com seis interações. Como reportado na Tabela 4, a classe dos antihipertensivos betabloqueadores foi a que apresentou as interações mais frequentes na população estudada. Dos pacientes com interações medicamentosas (moderadas ou altas), 43,3\% tinham no máximo duas interações e $10,3 \%$ apresentavam três ou mais interações.

\section{DISCUSSÃO}

Este estudo avaliou o uso dos medicamentos prescritos correlacionando os parâmetros de pressão arterial e dislipidemia com o entendimento da prescrição medicamentosa pela população idosa do SUS.

Os dados obtidos mostraram a prevalência de sexo feminino na população estudada. $O$ mesmo foi observado em outras pesquisas, como em Marques et al., ${ }^{25}$ onde o predomínio do sexo feminino foi de 90\% e em Arruda et al., ${ }^{12}$ que obteve a prevalência de $83 \%$ de mulheres. Essa diferença populacional pode ser justificada pelo maior número de mulheres residentes na cidade de Porto Alegre e também por elas apresentarem uma maior preocupação com a saúde e utilizarem mais os serviços de saúde. Além disso, o consumo de álcool e tabaco é inferior ao dos homens, determinando a maior expectativa de vida entre as mulheres. $., 26,27$

A maioria dos idosos estudados tinha entre 70 a 79 anos, e os pacientes que compreendiam a faixa etária de 80 anos ou mais apresentaram maior dificuldade no entendimento da prescrição. Isso pode estar relacionado, principalmente, a influência do envelhecimento na função cognitiva do idoso e ao esquecimento de fatos recentes. ${ }^{28}$

Os idosos atendidos no CEU-Vila Fátima demonstraram, significativamente, maior dificuldade no entendimento da prescrição médica, quando comparados aos idosos que frequentam o Ambulatório do IGG. Em outro estudo realizado com idosos de
Porto Alegre, 41,2\% dos pacientes entenderam a prescrição, ${ }^{26}$ sendo esse semelhante ao resultado encontrado no CEU-Vila Fátima, no presente estudo. Os níveis de escolaridade assim como a classe social podem ter forte influência no entendimento da prescrição médica. Uma pior condição econômica pode estar relacionada a uma pior condição de saúde resultando em esquemas terapêuticos mais complexos, havendo a necessidade de maior atenção por parte dos profissionais da saúde quanto à compreensão do tratamento por estes pacientes..$^{29}$ Blanski e Lenardt ${ }^{28}$ demonstraram que a maioria dos idosos estudados cursou da $1^{\text {a }}$ a $4^{\underline{a}}$ série $(44,4 \%)$ ou eram analfabetos $(22,2 \%)$, evidenciando que a escolaridade pode estar diretamente envolvida na compreensão e adesão ao tratamento medicamentoso.

Com relação à análise da pressão arterial dos pacientes estudados, observou-se o baixo índice de controle da mesma. Do total de pacientes (84) que fez uso de tratamento anti-hipertensivo, somente 30 pacientes estavam com a pressão arterial considerada controlada. Neste caso, não há evidências de que o mau controle da pressão arterial sofreu influências do não entendimento da prescrição. $O$ baixo índice no controle pode estar relacionado à falta de acesso aos medicamentos, ao esquecimento e a falta de esclarecimentos sobre os objetivos e importância do tratamento, já que se trata de uma doença silenciosa, sendo necessárias intervenções específicas aos pacientes de acordo com seus hábitos diários. ${ }^{30,31}$

$\mathrm{Na}$ análise do controle da dislipidemia, os resultados demonstraram uma tendência em relação ao entendimento da prescrição, assim sendo possível estimar que o entendimento da prescrição possa ser um fator importante para manter o perfil lipídico dentro dos limites recomendados. Dos pacientes atendidos no Ambulatório do IGG que mantêm o perfil lipídico controlado, 68,4\% entendem a prescrição. Porém, é importante ressaltar que estes exames podem ser interferidos pelo uso crônico de alguns medicamentos e/ou resultantes de interações medicamentosas. ${ }^{25}$ 
A polifarmácia favorece muitas interações medicamentosas, reações adversas e o surgimento de novos sintomas que aumentam o número de internações e danos à saúde. ${ }^{7}$ A elevada média de medicamentos prescritos em nosso estudo alerta tanto para os problemas já citados, como para a complexidade da terapia ao idoso, podendo interferir na adesão ao tratamento devido a dificuldades na administração dos mesmos e a falta de uma rede de apoio no tratamento.

Assim como no estudo desenvolvido por Schroeter et al. ${ }^{32}$ em que a média de medicamentos por paciente foi de $5,4 \pm 3,2$, observamos que o número de medicamentos independe do gênero. Mesmo que não significativo, é possível identificar a tendência ao aumento no número de medicamentos com a progressão da idade, como consequência do aumento da morbidade. ${ }^{32,33}$

As morbidades e as comorbidades também poderiam explicar a diferença significativa que foi observada entre os locais de estudo, já que a média de medicamentos prescritos aos pacientes atendidos no Ambulatório do IGG foi superior a média observada no CEU-Vila Fátima.

Com relação à hipertensão arterial, estudos têm revelado a preferência pelos diuréticos como tratamento anti-hipertensivo, tanto em monoterapias como em terapias combinadas. Assim como em nosso estudo, em que a classe dos diuréticos foi a mais utilizada, seguida pela classe dos inibidores da enzima conversora da angiotensina, pelos betabloqueadores e bloqueadores dos canais de cálcio. Trêset al., ${ }^{17}$ também identificou essas quatro classes como as mais prescritas, sendo a prevalência do uso de diuréticos em $81 \%$ dos tratamentos. Em outro estudo com pacientes idosos hipertensos de Porto Alegre, os resultados foram semelhantes, porém inferiores, relatando o uso dos diuréticos em $58,9 \%$ dos pacientes. ${ }^{30}$ A preferência pelo tratamento com diuréticos tiazídicospode ser devido ao seu baixo custo e da facilidade de aquisição pelo SUS, além de serem os fármacos indicados como tratamento de primeira escolha para monoterapias em idosos. ${ }^{17,34}$

Das interações medicamentosas observadas, as três mais freqüentes foram de gravidade moderada e envolveram, principalmente, medicamentos para o sistema cardiovascular. Foi identificada a interação entre biguanida (hipoglicemiantes orais) e betabloqueadores que podem aumentar o risco de desenvolver hiperglicemia ou hipoglicemia e/ou hipertensão. Com a mesma frequência, o uso concomitante de bloqueadores dos canais de cálcio e betabloqueadores pode ocasionar hipotensão e/ou bradicardia.
Seguida da interação entre anti-hipertensivos, diurético hidroclorotiazida e betabloqueador propranolol que podem interferir no perfil lipídico aumentando a glicemia e/ou os triglicerídeos. Em nosso estudo, somente dez pacientes apresentaram três ou mais interações medicamentosas, sendo quase metade dos pacientes $(46,4 \%)$ sem interações medicamentosas de gravidade moderada ou alta. Em contrapartida, outro estudo identificou interações clinicamente significantes em 90,6\% dos pacientes, porém essa diferença pode estar relacionada na metodologia utilizada para identificação das interações medicamentosas. ${ }^{35}$

A alta freqüência de interações medicamentosas envolvendo anti-hipertensivos pode ser devido ao número elevado de medicamentos prescritos para o sistema cardiovascular e, o mau controle tanto da hipertensão como da dislipidemia podem ser consequência dos efeitos dessas interações medicamentosas.

Uma análise posterior, dessa população, envolvendo a adesão correlacionada com os parâmetros laboratoriais analisados poderia reforçar nossos achados, verificando que o fato de entender a prescrição médica melhora a adesão à terapia medicamentosa.

Apesar de o estudo ter demonstrado quão importante é a orientação profissional, tanto sobre a doença como do tratamento medicamentoso, tivemos limitações como a falta de dados em prontuários, especialmente dos resultados laboratoriais de perfil lipídico e valores de pressão arterial. Os dados encontrados demonstram a importância do uso correto dos medicamentos e do entendimento da prescrição pelo paciente idoso. Para isso, é imprescindível que o paciente receba sua prescrição acompanhada de informações claras e simples sobre a importância de utilizar os medicamentos corretamente, além de esclarecimentos sobre as doenças que o acometem e as consequências que implicam o não uso dos mesmos. Os resultados obtidos devem servir como estímulo aos profissionais envolvidos na saúde do idoso, voltando-se a uma assistência multiprofissional e a prescrição racional, além do desenvolvimento de estratégias que facilitem a estes pacientes a administração de seus medicamentos.

\section{AGRADECIMENTOS}

Este estudo recebeu apoio do Conselho Nacional de Desenvolvimento Científico e Tecnológico (CNPq), número 475137/2009-4 e Projeto PRAIAS, PUCRS. Agradecemos ao Prof. Dr. Geraldo A. de Carli pela colaboração no projeto. 


\section{REFERÊNCIAS}

1. Organización Mundial de la Salud (OMS). Perspectivas políticas de la OMS sobre medicamentos Promoción del uso racional de medicamentos: componentes centrales. 2002. [Acesso em 16 jan 2017]. Disponível em: http://www. who.int/medicinedocs/collect/medicinedocs/pdf/s4874s s4874s.pdf.

2. Brasil. Ministério da Saúde. Política Nacional de Medicamentos. Brasília: Ministério da Saúde; 2001a.

3. World Health Organization (WHO). The rational use of drugs: report of the conference of experts. Nairobi 25-29 July 1985. Geneva: WHO; 1987.

4. Bucley BM. Healthy ageing: ageing safely. Eur Heart J Supplements. 2001;3:6-10.

5. Welker KL, Mycyk, MB. Pharmacology in the Geriatric Patient. Emerg Med Clin N Am. 2016;34:469-81.

6. Mclean AJ, Le Couteur DG. Aging Biology and Geriatric Clinical Pharmacology. Pharmacol Rev. 2004;56:163-84.

7. Oliveira LPBA, Santos SMA. Uma revisão integrativa sobre o uso de medicamentos por idosos na atenção primária à saúde. Rev Esc Enferm USP. 2016/50(1):167-79.

8. Novaes MRCG. Assistência farmacêutica ao idoso - uma abordagem multiprofissional. Brasília: Thesaurus; 2007. 244 p.

9. Morrone FB, Schroeter G, Petitembert AP, et al. Potential interactions of central nervous system drugs used in the elderly population. Brazilian J Pharm Sci. 2009;45(2): 227-34.

10. Venturini CD, Engroff P, Ely LS, et al. Gender differences, polypharmacy, and potential pharmacological interactions in the elderly. Clinics. 2011;66(11):1867-72.

11. Secoli SR. Polifarmácia: interações e reações adversas no uso de medicamentos por idosos. Rev Bras Enferm, Brasília. 2010;63(1):136-40.

12. Arruda DCJ, Eto FN, Velten APC, et al. Fatores associados a não adesão medicamentosa entre idosos de um ambulatório filantrópico do Espírito Santo. Rev Bras Geriatr Gerontol. 2015;18(2):327-37.

13. Radhamanohar M, Than M, Rizvi S. Assessment of patients' knowledge about their illness and treatment. Br J Clin Pract. 1993;47:23-5.

14. World Health Organization (WHO). Adherence to longterm therapies: evidence for action. Library Cataloguing-inPublication. 2003. [Acesso em 16 Jan 2017]. Disponível em: http://www.who.int/chp/knowledge/publications/adherence report/en/

15. Moreira RO, Santos RD, Martinez L, et al. Perfil Lipídico de pacientes com alto risco para Eventos Cardiovasculares na Prática Clínica Diária. Arq Bras Endocrinol Metab. $50(3): 481-9,2006$.

16. Sociedade Brasileira de Hipertensão (SBH). VI Diretrizes Brasileiras de Hipertensão Arterial, 2010. [Acesso em 19 Jan 2017]. Disponível em: http://publicacoes.cardiol.br/consenso/.

17. Três GS, Utzig JB, Martins R, et al. Controle da pressão arterial, do diabetes mellitus e da dislipidemia na população de hipertensos de um ambulatório de residência médica. Rev Bras Hipertens. 2009;16(3):143-7.

18. Linarelli MCB, Massarotto AC, Andrade AMCMG, et al. Análise do uso racional de medicamentos anti-hipertensivos utilizados em hospital-escola. Rev Ciênc Méd. 2009;18(4): 193-200.
19. De Paula JM. Entendimento da prescrição médica e melhora na atenção ao paciente diabético no Centro de Extensão Universitária. [Trabalho de Conclusão de Curso]. Porto Alegre: Pontifícia Universidade Católica do Rio Grande do Sul; 2007.

20. Folstein M, Folstein S. "Mini-Mental State" a practical method for grading the cognitive state of patients for the clinician. J. Psychiat. Res. 1975;12:189-98.

21. Brucki S, Nitrini R, Caramelli P, et al. Sugestões para o uso do Mini-Exame do estado mental no Brasil. Arq Neuropsiquiatr. 2003; (3-B):777-81.

22. DEF - Dicionário de Especialidades Farmacêuticas 2009/2010. Epub, 2009. 738 p.

23. World Health Organization (WHO). Collaborating Centre for Drug Statistics Methodology, Guidelines for ATC classification and DDD assignment 2010. 13 $3^{\text {th }}$ ed. Oslo, 2009.

24. III Diretrizes Brasileiras sobre Dislipidemias e Diretriz de Prevenção da Aterosclerose do Departamento de Aterosclerose da Sociedade Brasileira de Cardiologia. Ago 2001. [Acesso 14 dez. 2016]. Disponível em: http:// publicacoes.cardiol.br/consenso/

25. Marques LAM. Perfil lipídico de idosos atendidos por programa de Atenção Farmacêutica. Rev. Eletrônica de Extensão - UDESC em Ação. 2008;2(1):1-9.

26. Faggiani FT, Schroeter, G, Pacheco SL, et al. Profile of drug utilization in the elderly living in Porto Alegre, Brazil. Pharm Pract. 2007;5(4):179-84

27. Instituto Brasileiro de Geografia e Estatística (IBGE). Metodologia do censo demográfico 2000. Rio de Janeiro: IBGE; 2003. [Acessado 19 dez. 2016]. Disponível em: http:// www.ibge.gov.br/home/estatistica/populacao/censo2000/ metodologia/defaulttab.shtm.

28. Blanski CR, Lenardt MH. A compreensão da terapêutica medicamentosa pelo idoso. Rev Gaúcha Enferm. 2005; 26(2):180-8.

29. Acurcio FA, Silva AL, Ribeiro AQ, et al. Complexidade do regime terapêutico prescrito para idosos. Rev Assoc Med Bras. 2009;55(4):468-74.

30. Knorst D, Araujo BV. Atenção Farmacêutica em pacientes idosos hipertensos: uma experiência em Tucunduva-RS. Rev Bras Farm. 2008;89(4):290-3.

31. Abegaz TM, Shehab A, Gebreyohannes EA, et al. Nonadherence to antihypertensive drugs: A systematic review and meta-analysis. Medicine. 2017;96(4):e5641.

32. Schroeter G, Chaves LL, Engroff P, et al. Estudo De Utilização de Anti-Ulcerosos na população idosa de Porto Alegre, RS, Brasil. Rev HCPA. 2008;28(2):89-95.

33. Schroeter G, Trombetta T, Faggiani FT, et al. Terapia antihipertensiva utilizada por pacientes idosos de Porto Alegre/ RS, Brasil. Scientia Medica. 2007;17(1):14-9.

34. Miranda RD, Perrotti TC, Bellinazzi VR, et al. Hipertensão arterial no idoso: peculiaridades na fisiopatologia, no diagnóstico e no tratamento. Rev Bras Hipertens. 20072;9(3):293-300.

35. Basic-vrca V, Marusic S, Erdeljic V, et al. The incidence of potential drug-drug interactions in elderly patients with arterial hypertension. Pharm World Sci. 2010;32(6): 815-21. 